11,05

\title{
Магнитные фазовые переходы в несоизмеримую магнитную структуру в соединении $\mathrm{FeGe}_{2}$
}

\author{
(C) В.В. Меньшенин \\ Институт физики металлов им. М.Н. Михеева УрО РАН, \\ Екатеринбург, Россия \\ E-mail: menshenin@imp.uran.ru
}

(Поступила в Редакцию 1 октября 2018 г.)

На основе феноменологического рассмотрения в соединении $\mathrm{FeGe}_{2}$ проведен симметрийный анализ возможных магнитных структур в несоизмеримой магнитной фазе, появляющейся в результате фазовых переходов из парамагнитной фазы. Показано, что два возможных подхода к такому анализу, первый из которых использует магнитное представление пространственной группы, а второй опирается на разложение плотности магнитного момента по базисным функциям неприводимых представлений группы симметрии парамагнитной фазы, дают одни и те же результаты. Найдены неприводимые представления пространственной группы, по которым может происходить переход в несоизмеримую структуру. Набор этих представлений оказывается одинаковым в обоих подходах. Записаны функционалы Гинзбурга-Ландау для анализа переходов по этим представлениям. Проведен ренормгрупповой анализ фазовых переходов второго рода из парамагнитного состояния в несоизмеримую магнитную структуру. Показано, что спиральная магнитная структура может возникнуть в несоизмеримой фазе в результате двух фазовых переходов второго рода при одинаковой температуре переходов.

Работа выполнена в рамках бюджетного финансирования по теме „Квант“ Г.p. № АAАА-А18118020190095-4 и проекту № 18-2-2-11 Программы УрО РАН.

DOI: 10.21883/FTT.2019.03.47251.269

\section{1. Введение}

Открытие высокотемпературной сверхпроводимости в соединениях железа с sp-элементами $\mathrm{As}$, Te, $\mathrm{Se}$ вновь привлекло внимание исследователей к соединениям железа, обладающим слоистой структурой, поскольку в сверхпроводниках на основе FeAs-соединений, например, исходные недопированные соединения обладают слоистой структурой, а сами они являются металлическими антиферромагнетиками с температурой Нееля не выше $140 \mathrm{~K}[1]$.

Соединение $\mathrm{FeGe}_{2}$ также обладает слоистой структурой и показывает довольно необычные магнитные свойства. Из нейтронографических исследований [2-4], a также других данных $[5,6]$ установлено, что в этом соединении имеют место два магнитных перехода при температурах $T_{1} \approx 263 \mathrm{~K}$ и $T_{2} \approx 290 \mathrm{~K}$. До недавнего времени считалось, что при температурах ниже $263 \mathrm{~K}$ имеет место коллинеарное антиферромагнитное упорядочение. В промежутке между этими температурами в соединении возникает несоизмеримая магнитная структура. В недавней работе [7] из данных по нейтронному рассеянию в нулевом магнитном поле было найдено доказательство присутствия ферромагнитного порядка в преимущественно антиферромагнитной фазе при низких температурах. Нет единого взгляда на то, определяется ли магнитный порядок только свободными электронами, либо локализованные на ионах железа электроны также дают в клад в возникновение дальнего магнитного порядка. В работе [8] высказано утверждение, что маг- нитный порядок в антиферромагнитной фазе обусловлен только свободными электронами. Авторы работы [6] высказали предположение, что при температурах выше температуры $T_{1}$ подсистема локализованных электронов находится в разупорядоченном состоянии, в то время как ниже этой температуры эта подсистема имеет коллинеарную антиферромагнитную структуру с вектором антиферромагнетизма, лежащим в базисной плоскости кристалла. В подсистеме коллективизированных электронов при температурах ниже $T_{2}$ возникает несоизмеримая магнитная структура, которая сохраняется и при температуре ниже $T_{1}$.

Природа магнитных фазовых переходов при указанных выше температурах изучалась во многих работах. Авторы работы [3], исходя из нейтронографических данных, пришли к выводу, что магнитные переходы при этих температурах являются переходами второго рода, а магнитное упорядочение в несоизмеримой фазе является плоской спиралью с волновым вектором, ориентированным вдоль направления [100]. Однако в работах $[4,5,9]$ было установлено, что переход при температуре $T_{1}$ является переходом первого рода. Относительно природы фазового перехода при температуре $T_{2}$ также нет однозначной трактовки. Ренормгрупповой анализ этого перехода в работе [3], учитывающий только параметр порядка для структуры типа поперечной спиновой волны, привел авторов к заключению о том, что это переход второго рода. Опираясь далее на экспериментальные данные о том, что нужно принимать во внимание структуры не только типа поперечных, но и продольных 
спиновых волн, был проведен ренормгрупповой анализ перехода из парамагнитной в несоизмеримую фазу при учете двух типов структур. Оказалось, что этот переход может быть только первого рода. В работе [4] в рамках теории Ландау было найдено, что этот переход является переходом первого рода близким ко второму.

Отметим, что авторы работы [3] при записи функционалов Гинзбурга-Ландау обращали внимание только требования их вещественности, инвариантности относительно пространственной и временной инверсии. Трансформационные свойства параметра порядка, связанные группой симметрии соединения $\mathrm{FeGe}_{2}$, в рассмотрение не принимались. В работе [4] не учтено то обстоятельство, что для волнового вектора структуры с многолучевой звездой коэффициенты смешивания структуры, являющиеся компонентами параметра порядка, преобразуются по полному представлению пространственной группы [10].

Цель данной работы состоит в описании фазового перехода из парамагнитного состояния в несоизмеримую магнитную структуру в дигерманиде железа, базируясь на феноменологическом подходе и учитывая точно свойства симметрии параметров порядка рассматриваемого перехода. Использование феноменологического подхода позволяет не рассматривать микроскопические причины возникновения несоизмеримой структуры в этом соединении, которые могут являться темой отдельного исследования.

\section{2. Магнитные структуры $\mathrm{FeGe}_{2}$ в несоизмеримой фазе, допускаемые симметрией системы}

В работах $[11,12]$ указано, что кристаллическая симметрия парамагнитной фазы соединения $\mathrm{FeGe}_{2}$ характеризуется пространственной группой $I 4 / \mathrm{mcm}\left(D_{4 h}^{18}\right)$. Ионы железа занимают позицию $2 a$. Заметим, что далее везде работаем с примитивной ячейкой кристалла и рассматриваем для описания группы установку, данную в монографии Ковалева [13]. Векторы основных периодов примитивной ячейки равны

$$
\begin{aligned}
& \mathbf{a}_{1}=\left(\tau,-\tau, \tau_{z}\right)=(1,-1,1), \\
& \mathbf{a}_{2}=\left(-\tau, \tau, \tau_{z}\right)=(-1,1,1), \\
& \mathbf{a}_{3}=\left(\tau, \tau,-\tau_{z}\right)=(1,1,-1),
\end{aligned}
$$

где $2 \tau$ - длины периодов вдоль осей $X, Y$ системы координат, а $2 \tau_{z}$ - вдоль оси $Z$ этой системы. Вторые равенства в уравнениях (1) задают краткую запись основных периодов, в которой дальше приводятся нетривиальные трансляции элементов пространственной группы. Имеющее место при температуре $290 \mathrm{~K}$ возникновение дальнего магнитного порядка, несоизмеримого с периодами кристаллической решетки, не сопровождается изменением симметрии кристаллической решетки $[3,4]$. Из нейтронографических данных найдено, что волновой вектор магнитной структуры равен $\mathbf{k}=(2 \pi \mu / \tau, 0,0)$, $2 \mu \neq 0, \pm 1, \pm 2 \ldots$ В монографии Ковалева [13] это есть звезда $\mathbf{k}_{6}=\mu\left(\mathbf{b}_{2}+\mathbf{b}_{3}-\mathbf{b}_{1}\right)$, где $\mathbf{b}_{i}(i=1,2,3)-$ векторы обратной решетки. Звезда этого волнового вектора является четырехлучевой. Это означает, что при анализе возможной магнитной структуры необходимо принимать во внимание все четыре луча. Волновому вектору $\mathbf{k}$ соответствует четыре неприводимых представления пространственной группы, которые можно построить, зная неприводимые нагруженные представления группы волнового вектора, приведенные в монографии [13].

В работе [3] было установлено, что магнитная структура может быть хорошо описана, если рассматривать спиновые модуляции вдоль направлений [100] и [010]. Авторы этой работы утверждают, что эти модуляции представляют собой независимые поперечные или продольные волны спиновой плотности, распространяющиеся вдоль этих направлений, или плоскую спираль. В работе [4] высказано утверждение, что несоизмеримая магнитная структура характеризуется двумя неприводимыми представлениями, обозначаемыми в монографии [13] как $\tau_{2}$ и $\tau_{3}$. Это утверждение было основано на следующем обстоятельстве. При температуре $263 \mathrm{~K}$ система переходит в соизмеримую антиферромагнитную структуру с волновым вектором $\mathbf{k}=(2 \pi / \tau, 0,0)$ и описываемую неприводимым представлением $\tau_{9}$ пространственной группы $I 4 / \mathrm{mcm}\left(D_{4 h}^{18}\right)$ в результате фазового перехода первого рода. Это представление содержится в представлениях $\tau_{2}$ и $\tau_{3}$. Таким образом, эти два последних представления и будут связаны с несоизмеримой фазой соединения. Заметим, однако, что при фазовых переходах первого рода в общем случае нет подгрупповой связи между симметрией низкотемпературной и высокотемпературной фаз. Поэтому более последовательный, с нашей точки зрения, подход состоит в том, чтобы установить, какие представления содержатся в магнитном представлении [10] пространственной группы, а затем показать, что эти представления допускают существование структур типа спиновых волн, указанных выше.

Для определения характеров магнитного представления необходимо знать перестановки атомов железа при преобразованиях пространственной группы кристалла. Найдем эти перестановки только для элементов группы, которые оставляют волновой вектор $\mathbf{k}=(2 \pi \mu / \tau, 0,0)$ инвариантным. Эти элементы в представлении Вигнера-Зейтца имеют вид

$$
g_{2}=\left(h_{2} / 000\right), \quad g_{27}=\left(h_{27} / 001\right), \quad g_{28}=\left(h_{28} / 001\right),
$$

где $h_{2}$ - на $180^{\circ}$ вокруг оси $X, h_{27}=m_{y}-$ плоскость отражения, перпендикулярная оси $Y$, а $h_{28}=m_{z}-$ плоскость отражения, перпендикулярная оси $Z$, (001) нетривиальная трансляция вдоль главной оси кристалла в сокращенных обозначениях $(001)=\left(\mathbf{a}_{1}+\mathbf{a}_{2}\right) / 2$. Перестановки ионов железа приведены в табл. 1. 
Таблица 1. Перестановки атомов железа при действии элементов пространственной группы $I 4 / \mathrm{mcm}$

\begin{tabular}{c|c|c|c}
\hline $\begin{array}{c}\text { Номер атома } \\
\text { Возвращающая } \\
\text { трансляция }\end{array}$ & $h_{2}$ & $h_{27}$ & $h_{28}$ \\
\hline 1 & 1 & 2 & 3 \\
\hline $\mathbf{a}_{p}$ & $(000)$ & $(000)$ & $(000)$ \\
\hline 2 & 2 & 1 & 1 \\
$\mathbf{a}_{p}$ & $-\left(\mathbf{a}_{1}+\mathbf{a}_{2}\right)$ & $\left(\mathbf{a}_{1}+\mathbf{a}_{2}\right)$ & $(000)$
\end{tabular}

Таблица 2. Атомные магнитные моменты ионов железа для представлений $\tau_{2}$ и $\tau_{3}$

\begin{tabular}{c|c|c|c}
\hline Представление & Луч звезды & 1 & 2 \\
\hline$\tau_{2}$ & $\mathbf{k}$ & $(100)$ & $(100)$ \\
$\tau_{2}$ & $-\mathbf{k}$ & $(100)$ & $(100)$ \\
$\tau_{2}$ & $\mathbf{k}^{\prime}$ & $(010)$ & $(010)$ \\
$\tau_{2}$ & $-\mathbf{k}^{\prime}$ & $(010)$ & $(010)$ \\
$\tau_{3}$ & $\mathbf{k}$ & $(010)$ & $(010)$ \\
$\tau_{3}$ & $-\mathbf{k}$ & $(010)$ & $(010)$ \\
$\tau_{3}$ & $\mathbf{k}^{\prime}$ & $(-100)$ & $(-100)$ \\
$\tau_{3}$ & $-\mathbf{k}^{\prime}$ & $(-100)$ & $(-100)$ \\
$\tau_{3}^{\prime}$ & $\mathbf{k}$ & $(001)$ & $(001)$ \\
$\tau_{3}^{\prime}$ & $-\mathbf{k}$ & $(001)$ & $(001)$ \\
$\tau_{3}^{\prime}$ & $\mathbf{k}^{\prime}$ & $(001)$ & $(001)$ \\
$\tau_{3}^{\prime}$ & $-\mathbf{k}^{\prime}$ & $(001)$ & $(001)$
\end{tabular}

В табл. 1 вектор $\mathbf{a}_{p}$ - возвращающая трансляция, определение которой дано в [10]. Установим теперь, какие представления входят в состав магнитного представления. Можно легко показать, используя данные табл. 1 и выражение для характера магнитного представления, приведенное в [10], что

$$
\chi_{m}^{k_{6}}(E)=6, \chi_{m}^{k_{6}}\left(g_{2}\right)=-2, \quad \chi_{m}^{k_{6}}\left(g_{27}\right)=0, \chi_{m}^{k_{6}}\left(g_{28}\right)=0 .
$$

Здесь $E$ - тождественное преобразование, $\chi_{m}^{k_{6}}(g)-$ характер элемента $g$ пространственной группы для магнитного представления. С помощью стандартной процедуры [14] определяем, что из четырех представлений $\tau_{i}$, $i=1, \ldots, 4$ представление $\tau_{2}$ содержится в магнитном представлении один раз, а представление $\tau_{3}$ дважды, представление $\tau_{4}$ также два раза.

Используя теперь выражение для атомных магнитных моментов, приведенное в [10], найдем эти моменты для дигерманида железа. Они приведены в табл. 2.

В табл. 2 волновой вектор $\mathbf{k}^{\prime}=(0,2 \pi \mu / \tau, 0)$. Отметим, что выписанные в табл. 2 атомные магнитные моменты для представлений $\tau_{2}$ и $\tau_{3}$ совпадают с их значениями для атомов 1 и 2 в работе [4]. Значения этих моментов для представления $\tau_{3}^{\prime}$ в этой работе вообще не включалось в рассмотрение.
Представление $\tau_{2}$ допускает в несоизмеримой фазе следующее выражение для магнитного момента на узле, описываемом трансляцией $\mathbf{t}=n_{1} \mathbf{a}_{1}+n_{2} \mathbf{a}_{2}+n_{3} \mathbf{a}_{3}$, где $n_{i}$ $(i=1,2,3)$ - произвольные целые числа

$$
\begin{aligned}
& \eta_{1}\left(\begin{array}{l}
1 \\
0 \\
0
\end{array}\right) \exp (i \mathbf{k t})+\eta_{2}\left(\begin{array}{l}
1 \\
0 \\
0
\end{array}\right) \exp (-i \mathbf{k t}) \\
& \quad+\eta_{3}\left(\begin{array}{l}
0 \\
1 \\
0
\end{array}\right) \exp \left(i \mathbf{k}^{\prime} \mathbf{t}\right)+\eta_{4}\left(\begin{array}{l}
0 \\
1 \\
0
\end{array}\right) \exp \left(-i \mathbf{k}^{\prime} \mathbf{t}\right) .
\end{aligned}
$$

В последнем равенстве величины $\eta_{j}(j=1, \ldots, 4)$ представляют собой коэффициенты смешивания и могут рассматриваться как компоненты параметра порядка. Исходя из условия, что магнитный момент (4) должен быть действительной величиной, положим $\eta_{1}=\eta_{2}$, $\eta_{3}=\eta_{4}$. В результате имеем значения компонент для атомного магнитного момента на узле $\left\{n_{1}, n_{2}, n_{3}\right\}$

$$
\left(\begin{array}{l}
2 \eta_{1} \cos \left(\left[-n_{1}+n_{2}+n_{3}\right] 2 \pi \mu\right) \\
2 \eta_{3} \cos \left(\left[n_{1}-n_{2}+n_{3}\right] 2 \pi \mu\right)
\end{array}\right) .
$$

Выражения (4) и (5) показывают, что магнитная структура представляет собой два типа доменов структур типа продольной спиновой волны с волновыми векторами $\mathbf{k}$ и $\mathbf{k}^{\prime}$ соответственно. Таким образом, представление $\tau_{2}$ связано со структурами типа продольной спиновой волны.

Аналогичным образом для представления $\tau_{3}$ получим общее выражение для магнитного момента на узле $\left\{n_{1}, n_{2}, n_{3}\right\}$

$$
\begin{aligned}
& \zeta_{1}\left(\begin{array}{l}
0 \\
1 \\
0
\end{array}\right) \exp (i \mathbf{k t})+\xi_{2}\left(\begin{array}{l}
0 \\
1 \\
0
\end{array}\right) \exp (-i \mathbf{k t}) \\
& +\xi_{3}\left(\begin{array}{l}
\overline{1} \\
0 \\
0
\end{array}\right) \exp \left(i \mathbf{k}^{\prime} \mathbf{t}\right)+\xi_{4}\left(\begin{array}{l}
\overline{1} \\
0 \\
0
\end{array}\right) \exp \left(-i \mathbf{k}^{\prime} \mathbf{t}\right) .
\end{aligned}
$$

Снова используя условие вещественности атомного магнитного момента, имеем равенства $\xi_{1}=\xi_{2}, \xi_{3}=\xi_{4}$, а его компоненты на узле $\left\{n_{1}, n_{2}, n_{3}\right\}$ имеет вид

$$
\left(\begin{array}{r}
-2 \xi_{3} \cos \left(\left[\left[n_{1}-n_{2}+n_{3}\right] 2 \pi \mu\right]\right) \\
2 \xi_{1} \cos \left(\left[-n_{1}+n_{2}+n_{3}\right] 2 \pi \mu\right)
\end{array}\right) .
$$

Выражения (6), (7) показывают, что представление $\tau_{3}$ связано со структурами типа поперечной спиновой волны, поскольку магнитная структура представляет собой снова два домена таких волн с волновыми векторами $\mathbf{k}$ и $\mathbf{k}^{\prime}$ соответственно. Мы не будем останавливаться сейчас на представлениях $\tau_{3}^{\prime}, \tau_{4}, \tau_{4}^{\prime}$, поскольку они будут рассмотрены дальше несколько иным способом.

Таким образом, мы показали, что представления $\tau_{2}, \tau_{3}$ действительно связаны с магнитными несоизмеримыми структурами, которые были предложены в работе [3], исходя из нейтронографических данных. 


\section{3. Полные неприводимые представления}

Подойдем к анализу возможных магнитных структур несколько иным способом, предложенным ранее в работах Ковалева и Дзялошинского [15,16]. Начнем рассмотрение с разложения плотности магнитного момента кристалла $\mathbf{M}(\mathbf{r})$ в несоизмеримой фазе по базисным функциям неприводимых представлений пространственной группы парамагнитной фазы $G \times \overline{1}$, где $\overline{1}-$ пространственная инверсия. Это разложение имеет вид

$$
\mathbf{M}(\mathbf{r})=\sum_{i, \alpha, n} c_{i \alpha n} \mathbf{s}_{\alpha} \varphi_{i n}
$$

где $\varphi_{i n}-$ базисные функции неприводимого представления, характеризуемого номером $n$, индекс $i$ нумерует базисные функции представления, $\mathbf{s}_{\alpha}-$ псевдовекторные ортогональные орты, $c_{i \alpha n}-$ коэффициенты разложения. В дальнейшем вместо группы $G \times \overline{1}-$ рассматривается группа $G$ и принимается во внимание, что компоненты плотности магнитного момента могут входить в выражение для термодинамического потенциала только в четных степенях. Волновому вектору $\mathbf{k}$, приведенному выше, соответствует в группе $I 4 / \mathrm{mcm}\left(D_{4 h}^{18}\right)$ четыре неприводимых представления полной пространственной группы [14].

Представление полной пространственной группы, связанное с $\tau_{1}$, описывается следующими матрицами

$$
\begin{aligned}
& D^{\{k\} \tau_{1}}\left(g_{2}\right)=\left[\begin{array}{cccc}
1 & 0 & 0 & 0 \\
0 & 1 & 0 & 0 \\
0 & 0 & 0 & 1 \\
0 & 0 & 1 & 0
\end{array}\right] ; \quad D^{\{k\} \tau_{1}}\left(g_{14}\right)=\left[\begin{array}{cccc}
0 & 0 & 0 & 1 \\
0 & 0 & 1 & 0 \\
1 & 0 & 0 & 0 \\
0 & 1 & 0 & 0
\end{array}\right] ; \\
& D^{\{k\} \tau_{1}}\left(g_{25}\right)=\left[\begin{array}{cccc}
0 & 1 & 0 & 0 \\
1 & 0 & 0 & 0 \\
0 & 0 & 0 & 1 \\
0 & 0 & 1 & 0
\end{array}\right] \text {; }
\end{aligned}
$$

В равенствах (9) $g_{14}=\left(h_{14} / 000\right), g_{25}=\left(h_{25} / 001\right), h_{14}-$ поворот на $90^{\circ}$ вокруг оси $Z, h_{25}$ - пространственная инверсия. Матричное представление $D^{\{k\}, \tau_{1}}$ для остальных элементов группы получается в результате перемножений этих матриц с учетом нетривиальных трансляций. Представление $\tau_{2}$ приводит к следующему представлению полной пространственной группы

$$
\begin{aligned}
& D^{\{k\} \tau_{2}}\left(g_{2}\right)=\left[\begin{array}{cccc}
1 & 0 & 0 & 0 \\
0 & 1 & 0 & 0 \\
0 & 0 & 0 & 1 \\
0 & 0 & 1 & 0
\end{array}\right], D^{\{k\} \tau_{2}}\left(g_{14}\right)=\left[\begin{array}{cccc}
0 & 0 & 0 & 1 \\
0 & 0 & 1 & 0 \\
1 & 0 & 0 & 0 \\
0 & 1 & 0 & 0
\end{array}\right], \\
& D^{\{k\} \tau_{2}}\left(g_{25}\right)=\left[\begin{array}{rrrr}
0 & -1 & 0 & 0 \\
-1 & 0 & 0 & 0 \\
0 & 0 & 0 & -1 \\
0 & 0 & -1 & 0
\end{array}\right] \text {. }
\end{aligned}
$$

Соответственно для представления $\tau_{3}$ имеем матричное представление

$$
\begin{aligned}
& D^{\{k\} \tau_{3}}\left(g_{2}\right)=\left[\begin{array}{rrrr}
-1 & 0 & 0 & 0 \\
0 & -1 & 0 & 0 \\
0 & 0 & 0 & -1 \\
0 & 0 & -1 & 0
\end{array}\right], \\
& D^{\{k\} \tau_{3}}\left(g_{14}\right)=\left[\begin{array}{rrrr}
0 & 0 & 0 & -1 \\
0 & 0 & 1 & 0 \\
-1 & 0 & 0 & 0 \\
0 & 1 & 0 & 0
\end{array}\right],
\end{aligned}
$$

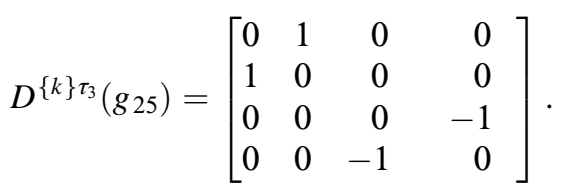

Представление $\tau_{4}$ приводит к представлению полной пространственной группы в виде

$$
\begin{aligned}
& D^{\{k\} \tau_{4}}\left(g_{2}\right)=\left[\begin{array}{rrrr}
-1 & 0 & 0 & 0 \\
0 & -1 & 0 & 0 \\
0 & 0 & 0 & -1 \\
0 & 0 & -1 & 0
\end{array}\right], \\
& D^{\{k\} \tau_{4}}\left(g_{14}\right)=\left[\begin{array}{rrrr}
0 & 0 & 0 & -1 \\
0 & 0 & 1 & 0 \\
-1 & 0 & 0 & 0 \\
0 & 1 & 0 & 0
\end{array}\right] \text {, } \\
& D^{\{k\} \tau_{4}}\left(g_{25}\right)=\left[\begin{array}{rrrr}
0 & -1 & 0 & 0 \\
-1 & 0 & 0 & 0 \\
0 & 0 & 0 & 1 \\
0 & 0 & 1 & 0
\end{array}\right] .
\end{aligned}
$$

Базисные функции неприводимых представлений пространственных групп могут быть выбраны в виде функций Блоха $\varphi(\mathbf{r})=u_{k}(\mathbf{r}) \exp (i \mathbf{k r})$. Эти функции под действием элемента пространственной группы $(h / \tau)$ преобразуются следующим образом [17]

$$
(h / \boldsymbol{\tau}) \varphi(\mathbf{r})=\varphi\left(h^{-1}(\mathbf{r}-\boldsymbol{\tau})\right) .
$$

Используя равенства (13) и (9) просто показать, что базисные функции представления $D^{\{k\} \tau_{1}}$ имеют следующий вид

$$
\begin{array}{ll}
\varphi_{1 \tau_{1}}=A \exp (i 2 \pi \mu x / \tau), & \varphi_{2 \tau_{1}}=A \exp (-i 2 \pi \mu x / \tau), \\
\varphi_{3 \tau_{1}}=A \exp (i 2 \pi \mu y / \tau), & \varphi_{4 \tau_{1}}=A \exp (-i 2 \pi \tau y / \tau),
\end{array}
$$

где $A-$ некоторая константа, $2 \pi \mu x / \tau=\mathbf{k r}, 2 \pi \mu y / \tau=\mathbf{k}^{\prime} \mathbf{r}$. Базисные функции неприводимого представления $D^{\{k\} \tau_{2}}$ записываются следующим образом

$$
\begin{aligned}
\varphi_{1 \tau_{2}} & =\cos \left(\pi z / \tau_{z}\right) \exp \{i 2 \pi \mu x / \tau\}, \\
\varphi_{2 \tau_{2}} & =\cos \left(\pi z / \tau_{z}\right) \exp \{-i 2 \pi \mu x / \tau\}, \\
\varphi_{3 \tau_{2}} & =\cos \left(\pi z / \tau_{z}\right) \exp \{i 2 \pi \mu y / \tau\}, \\
\varphi_{4 \tau_{2}} & =\cos \left(\pi z / \tau_{z}\right) \exp \{-i 2 \pi \mu y / \tau\} .
\end{aligned}
$$


Мы не будем приводить здесь явно базисные функции для представлений $\tau_{3}, \tau_{4}$. Отметим лишь, что базисные функции этих представлений содержат множители $\sin (\pi x / \tau)$ или $\sin (\pi y / \tau)$, которые в начале координат обращаются в нуль. Одно из важных ограничений на то, базисные функции каких представлений участвуют в разложении (8), состоит в том, что эта спиновая плотность должна быть отлична от нуля в позициях магнитных ионов [15]. Поскольку координаты первого атома в нашем случае равны нулю, то представления $\tau_{3}, \tau_{4}$ не входят в выражение (8). Более формальным способом этот результат можно получить методом, предложенным в работе [15].

Совокупность величин, представляющих собой произведение аксиальных ортов и указанных выше функций, соответствующих одному неприводимому представлению, преобразуется по прямому произведению этого неприводимого представления пространственной группы на векторное аксиальное представление. Это представление приводимо и его можно разложить по неприводимым представлениям пространственной группы. Можно просто показать, аналогично работе [15], что представление $V^{\prime}(g) \otimes D^{\{k\} \tau_{1}}$, где $V^{\prime}(g)$ - псевдовекторное представление, разбивается на представления $D^{\{k\} \tau_{2}}, D^{\{k\} \tau_{3}}$, $D^{\{k\} \tau_{4}}$, тогда как представление $V^{\prime}(g) \otimes D^{\{k\} \tau_{2}}$ разлагается на $D^{\{k\} \tau_{1}}, D^{\{k\} \tau_{3}}, D^{\{k\} \tau_{4}}$. Поскольку не все элементы пространственной группы оставляют неизменными позиции первого и второго атомов, то представление $D^{\{k\} \tau_{1}}$ в этом разложении может быть опущено. Заметим, что полученный только что результат полностью совпадает с найденным из разложения на неприводимые представления магнитного представления пространственной группы. Именно по этим неприводимым представлениям должны происходить фазовые переходы в несоизмеримую структуру. Используя метод проекционных операторов, определим, исходя из базисных функций представлений $V^{\prime}(g) \otimes D^{\{k\} \tau_{1}}$ и $V^{\prime}(g) \otimes D^{\{k\} \tau_{2}}$, базисные функции тех представлений, на которые последние разлагаются. Эти базисные функции для представлений $D^{\{k\} \tau_{2}}, D^{\{k\} \tau_{3}}, D^{\{k\} \tau_{4}}$ запишутся в виде

$$
\begin{aligned}
& \psi_{1}^{\tau_{2}}=s_{x} A \exp (i 2 \pi \mu x / \tau), \quad \psi_{2}^{\tau_{2}}=-s_{x} A \exp (-i 2 \pi \mu x / \tau), \\
& \psi_{3}^{\tau_{2}}=s_{y} A \exp (i 2 \pi \mu y / \tau), \quad \psi_{4}^{\tau_{2}}=-s_{y} A \exp (-i 2 \pi \mu y / \tau),
\end{aligned}
$$

$$
\begin{gathered}
\psi_{1}^{\tau_{3}}=s_{y} A \exp (i 2 \pi \mu x / \tau), \quad \psi_{2}^{\tau_{3}}=s_{y} A \exp (-i 2 \pi \mu x / \tau), \\
\psi_{3}^{\tau_{3}}=s_{x} A \exp (i 2 \pi \mu y / \tau), \quad \psi_{4}^{\tau_{3}}=-s_{x} A \exp (-i 2 \pi \mu y / \tau),
\end{gathered}
$$

$$
\psi_{1}^{\tau_{4}}=s_{z} A \exp (i 2 \pi \mu x / \tau), \quad \psi_{2}^{\tau_{4}}=-s_{z} A \exp (-i 2 \pi \mu x / \tau),
$$$$
\psi_{3}^{\tau_{4}}=-s_{z} A \exp (i 2 \pi \mu y / \tau), \quad \psi_{4}^{\tau_{4}}=-s_{z} A \exp (-i 2 \pi \mu y / \tau) .
$$

Базисные функции представлений $D^{\{k\} \tau_{3}}, D^{\{k\} \tau_{4}}$, участвующие в разложении $V^{\prime}(g) \otimes D^{\{k\} \tau_{2}}$, можно запи- сать следующим образом

$$
\begin{aligned}
& \psi_{1}^{\prime \tau_{3}}=s_{z} \cos \left(\pi z / \tau_{z}\right) \exp (i 2 \pi \mu x / \tau), \\
& \psi_{2}^{\prime \tau_{3}}=-s_{z} \cos \left(\pi z / \tau_{z}\right) \exp (-i 2 \pi \mu x / \tau), \\
& \psi_{3}^{\prime \tau_{3}}=-s_{z} \cos \left(\pi z / \tau_{z}\right) \exp (i 2 \pi \mu y / \tau), \\
& \psi_{4}^{\prime \tau_{3}}=-s_{z} \cos \left(\pi z / \tau_{z}\right) \exp (-i 2 \pi \mu y / \tau), \\
& \psi_{1}^{\prime \tau_{4}}=s_{y} \cos \left(\pi z / \tau_{z}\right) \exp (i 2 \pi \mu x / \tau), \\
& \psi_{2}^{\prime \tau_{4}}=s_{y} \cos \left(\pi z / \tau_{z}\right) \exp (-i 2 \pi \mu x / \tau), \\
& \psi_{3}^{\prime \tau_{4}}=s_{x} \cos \left(\pi z / \tau_{z}\right) \exp (i 2 \pi \mu y / \tau), \\
& \psi_{4}^{\prime \tau_{4}}=-s_{x} \cos \left(\pi z / \tau_{z}\right) \exp (-i 2 \pi \mu y / \tau) .
\end{aligned}
$$

Выражение для спиновой плотности (8) после разложения представлений $V^{\prime}(g) \otimes D^{\{k\} \tau_{1}}$ и $V^{\prime}(g) \otimes D^{\{k\} \tau_{2}}$ на неприводимые представления должно записываться через базисные функции $(16)-(20)$ этих неприводимых представлений. Это выражение имеет вид

$$
\mathbf{M}(\mathbf{r})=\sum_{i, n} \eta_{i n} \psi_{i}^{\tau_{n}}+\sum_{j, m} \eta_{j m}^{\prime} \psi_{j}^{\prime \tau_{m}},
$$

где в первой сумме индексы $i$ и $n$ принимают значения $i=1, \ldots, 4, n=2,3,4$, а во второй сумме $j=1, \ldots, 4$, $m=3,4$. Из этого выражения становится ясно, что разложения (4) и (6) с точностью до обозначения констант совпадают с вкладом от представлений $\tau_{2}, \tau_{3}$ в первую сумму в выражении (21), поскольку проекция первого орта в (8) на ось $x$ равна $s_{x}=(1,0,0)$, а второго орта на ось $y$ есть $s_{y}=(0,1,0)$. Таким образом, представления $D^{\{k\} \tau_{2}}, D^{\{k\} \tau_{3}}$ с базисными функциями (16) и (17) определяют продольные и поперечные волны спиновой плотности соответственно. Представление $D^{\{k\} \tau_{4}}$ с базисными функциями (18) задает волны поперечной спиновой плотности, в которых плотность магнитного момента ориентирована вдоль оси $z$ кристалла. Эту же ориентацию момента в поперечных волнах спиновой плотности задает представление $D^{\{k\} \tau_{3}}$ с базисными функциями (19). Представление $D^{\{k\} \tau_{4}}$ с базисными функциями (20) определяет волны поперечной спиновой плотности. Отметим. что из нейтронографических исследований, результаты которых приведены в работе [3], следует, что плотность спинового момента лежит в плоскости $X Y$ системы.

\section{4. Эффективный гамильтониан}

Остановимся теперь на переходе в несоизмеримую фазу в соединении $\mathrm{FeGe}_{2}$. Одно из основных положений теории фазовых переходов Ландау состоит в том, что фазовый переход второго рода происходит по единственному представлению, а переход по двум представлениям возможен только в изолированной точке плоскости $(P, T$, где $P-$ давление, $T-$ температура), которая является пересечением двух линий фазовых переходов 
второго рода [18]. Запишем поэтому для начала эффективный гамильтониан для перехода по представлению $\tau_{2}$, характеризуемому НП пространственной группы $D^{\{k\} \tau_{2}}$.

Исходя из правил преобразования функций $\psi_{i}^{\tau_{2}}(16)$ под действием элементов пространственной группы, задаваемых матрицами $D^{\{k\} \tau_{2}}(10)$, найдем, что имеются следующие инвариантные комбинации, квадратичные по этим полям

$$
\begin{gathered}
I_{1}=\left(\psi_{1}^{\tau_{2}}+\psi_{2}^{\tau_{2}}\right)^{2}+\left(\psi_{3}^{\tau_{2}}+\psi_{4}^{\tau_{2}}\right)^{2}, \\
I_{2}=\left(\psi_{1}^{\tau_{2}}-\psi_{2}^{\tau_{2}}\right)^{2}+\left(\psi_{3}^{\tau_{2}}-\psi_{4}^{\tau_{2}}\right)^{2}, \\
I_{3}=\psi_{1}^{\tau_{2}} \psi_{2}^{\tau_{2}}+\psi_{3}^{\tau_{2}} \psi_{4}^{\tau_{2}}=-\frac{1}{2}\left(\left|\psi_{1}^{\tau_{2}}\right|^{2}+\left|\psi_{3}^{\tau_{2}}\right|^{2}+\left|\psi_{2}^{\tau_{2}}\right|^{2}+\left|\psi_{4}^{\tau_{2}}\right|^{2}\right) .
\end{gathered}
$$

Эти инварианты не являются независимыми, так как $I_{1}-I_{2}=4 I_{3}$. Поэтому в качестве независимого выберем инвариант $I_{3}$. Введем обозначения

$$
\begin{array}{ll}
\psi_{1}^{\tau_{2}}=\phi_{1}+i \bar{\phi}_{1}, & \psi_{2}^{\tau_{2}}=i \bar{\phi}_{1}-\phi_{1}, \\
\psi_{3}^{\tau_{2}}=\phi_{2}+i \bar{\phi}_{2}, & \psi_{4}^{\tau_{2}}=i \bar{\phi}_{2}-\phi_{2} .
\end{array}
$$

В этих обозначениях для указанного инварианта получим выражение

$$
I_{3}=-\left(\phi_{1}^{2}+\bar{\phi}_{1}^{2}+\phi_{2}^{2}+\bar{\phi}_{2}^{2}\right) .
$$

Просто показать, полагая постоянную $A$ равной единице, что выполняются равенства $\phi_{1}^{2}+\bar{\phi}_{1}^{2}=s_{x}^{2}$, a $\phi_{2}^{2}+\bar{\phi}_{2}^{2}=s_{y}^{2}$, Отсюда следует, что инвариант $I_{3}=-\left(s_{x}^{2}+s_{y}^{2}\right)$. Величины $s_{x}, s_{y}$ могут рассматриваться теперь как компоненты двумерного вектора, то есть этот инвариант имеет обменную природу, поскольку квадрат длины этого вектора не меняется при спиновых поворотах на любой угол вокруг любой оси. Другим указанием на то, что этот инвариант оказывается обменной природы является правая часть равенства (22), представляющая собой с точностью до постоянной сумму квадратов модулей базисных функций, которая не меняется при любых преобразованиях. Покажем, что и в подходе Дзялошинского инвариант аналогичный $I_{3}$ приводит к обменному члену. Запишем для этого, следуя [16] и используя базисные функции (14), следующие величины

$$
\mathbf{S}_{1 i}=\left(s_{x} \phi_{i \tau_{1}}, s_{y} \phi_{i \tau_{1}}, s_{z} \phi_{i \tau_{1}}\right), \quad i=1, \ldots, 4,
$$

где $\mathbf{S}=\left(s_{x}, s_{y}, s_{z}\right)-$ спиновый вектор, а постоянную $A$ в базисных функциях положим равной единице. Обменный инвариант равен $2 \mathbf{S}^{2}=\left(\mathbf{S}_{11} \mathbf{S}_{12}+\mathbf{S}_{13} \mathbf{S}_{14}\right)$, то есть эквивалентен инварианту $I_{3}$. Отметим, что в работе [16] обменный инвариант второго порядка записывался в виде

$$
\begin{aligned}
\mathbf{S}_{1 i} \mathbf{S}_{1 i} & =2 \mathbf{S}^{2}\left(\cos \frac{4 \pi \mu x}{\tau}+\cos \frac{4 \pi \mu y}{\tau}\right) \\
& =S^{2}\left(\varphi_{1 \tau_{1}}^{2}+\varphi_{2 \tau_{1}}^{2}+\varphi_{3 \tau_{1}}^{2}+\varphi_{4 \tau_{1}}^{2}\right) .
\end{aligned}
$$

Эта величина действительно инвариантна относительно чисто спиновых вращений, но содержит также и пространственный вклад. Сами величины $\mathbf{S}_{1 \alpha}(\alpha=1, \ldots, 4)$ преобразуются по представлению $V^{\prime}(g) \otimes D^{\{k\} \tau_{1}}$. Поэтому величина (26) инвариантна также относительно преобразований последнего представления. Инвариантной величиной относительно этого представления и имеющей чисто обменный характер является именно $2 \mathbf{S}^{2}=\left(\mathbf{S}_{11} \mathbf{S}_{12}+\mathbf{S}_{13} \mathbf{S}_{14}\right)$. Этот скаляр инвариантен как относительно спиновых вращений, так и пространственных преобразований, поскольку в разложении (25) $\mathbf{S}$ постоянный спиновый вектор единичной длины.

Преимущество развиваемого подхода с использованием базисных функций неприводимых представлений состоит в данном случае в том, что удается исключить из рассмотрения $s_{z}$ компоненту спина, которая в этом представлении отсутствует из требований симметрии. Инварианты четвертого порядка равны

$$
I_{3}^{2}=\left(s_{x}^{2}+s_{y}^{2}\right)^{2}, \quad I_{4}=\psi_{1}^{\tau_{2}} \psi_{2}^{\tau_{2}} \psi_{3}^{\tau_{2}} \psi_{4}^{\tau_{2}}=s_{x}^{2} s_{y}^{2} .
$$

Взаимодействие магнитных моментов, связанное с неоднородным распределением спиновой плотности, описывается инвариантом вида

$$
\begin{aligned}
I_{e x}^{\tau_{2}} & =\frac{\partial \psi_{1}^{\tau_{2}}}{\partial x_{i}} \frac{\partial \psi_{2}^{\tau_{2}}}{\partial x_{i}}+\frac{\partial \psi_{3}^{\tau_{2}}}{\partial x_{i}} \frac{\partial \psi_{4}^{\tau_{2}}}{\partial x_{i}} \\
& =\frac{\partial s_{x}}{\partial x_{i}} \frac{\partial s_{x}}{\partial x_{i}}+\frac{\partial s_{y}}{\partial x_{i}} \frac{\partial s_{y}}{\partial x_{i}}+\left(k_{x}^{2} s_{x}^{2}+k_{y}^{\prime 2} s_{y}^{2}\right),
\end{aligned}
$$

где по повторяющимся индексам подразумевается суммирование, а индекс $i=1,2,3$. Здесь и далее мы полагаем, что величины $s_{x}, s_{y}$ могут зависеть от всех пространственных координат. В этом выражении все слагаемые в правой части равенства (28) имеют обменную природу. Вблизи точки фазового перехода последний член $\left(k_{x}^{2} s_{x}^{2}+k_{y}^{\prime 2} s_{y}^{2}\right)$ приводит к перенормировке константы в эффективном гамильтониане и может быть опущен. Отметим теперь, что волновой вектор магнитной структуры имеет группу симметрии $C_{2 v}$. Поэтому переход второго рода может реализоваться только в специальном случае малости инварианта Лифшица, по сравнению со слагаемыми неоднородного обмена в эффективном гамильтониане [16]. Инвариант Лифшица может быть записан в рассматриваемой ситуации в виде

$$
I_{L}=\frac{\partial \psi_{1}^{\tau_{2}}}{\partial x} \psi_{2}^{\tau_{2}}-\psi_{1}^{\tau_{2}} \frac{\partial \psi_{2}^{\tau_{2}}}{\partial x}+\frac{\partial \psi_{3}^{\tau_{2}}}{\partial y} \psi_{4}^{\tau_{2}}-\psi_{3}^{\tau_{2}} \frac{\partial \psi_{4}^{\tau_{2}}}{\partial y} .
$$

Подставляя в выражение для инварианта Лифшица равенства (16) найдем, что этот инвариант равен

$$
I_{L}=-i\left(k_{x} s_{x}^{2}+k_{y}^{\prime} s_{y}^{2}\right)=-i\left(\mathbf{k} \mathbf{s} s_{x}+\mathbf{k}^{\prime} \mathbf{s} s_{y}\right) .
$$

Из вида этого выражения заключаем, что инвариант Лифшица в рассматриваемом случае имеет релятивистский характер, поскольку при преобразованиях симметрии преобразуются как компоненты волновых векторов, 
так и спиновые компоненты. Поэтому при исследовании поведения системы в окрестности точки фазового перехода, где существенны спиновые флуктуации, инвариантом Лифшица можно пренебречь.

Эффективный гамильтониан для описания фазового перехода в длиннопериодическую структуру с учетом инвариантов $I_{3}, I_{3}^{2}, I_{4}, I_{e x}^{\tau_{2}}$ имеет вид в области развитых спиновых флуктуаций

$$
\begin{aligned}
H_{e f f}^{\tau_{2}}= & \int d^{d} x\left\{\frac{r_{1}}{2}\left(s_{x}^{2}+s_{y}^{2}\right)+u_{1}\left(s_{x}^{2}+s_{y}^{2}\right)^{2}\right. \\
& \left.+u_{2} s_{x}^{2} s_{y}^{2}+\frac{1}{2}\left[\left(\frac{\partial s_{x}}{\partial x_{i}}\right)^{2}+\left(\frac{\partial s_{y}}{\partial x_{i}}\right)^{2}\right]\right\},
\end{aligned}
$$

где $d=4-\varepsilon$ размерность пространства $\varepsilon \ll 1$. Выражение (31) аналогично выражению (5) работы [3]. Однако в нашем случае эффективный гамильтониан описывает переход в структуру с волнами продольной, а не поперечной спиновой плотности.

Покажем теперь, принимая во внимание тот факт, что коэффициенты $\eta_{i 2} i=1, \ldots, 4$, а не базисные функции в первом слагаемом в равенстве (21), можно считать преобразующимися по неприводимому представлению (10), можно получить эффективный гамильтониан, имеющий вид (31). Учитывая свойства компонент $\eta_{i 2}$ $(i=1, \ldots, 4)$ при преобразованиях (10), введем переменные

$$
\eta_{1 r}=\eta_{12}+\eta_{22}, \quad \eta_{2 r}=\eta_{32}+\eta_{42} .
$$

Тогда эффективный гамильтониан, описывающий переход по этому представлению, может быть записан в виде

$$
\begin{aligned}
H_{e f f}^{\tau_{2}} & =\int d^{d} x\left(\frac{1}{2}\left[\left(\frac{\partial \eta_{1 r}}{\partial x}\right)^{2}+\left(\frac{\partial \eta_{2 r}}{\partial x}\right)^{2}\right]+\frac{r_{1}}{2}\left(\eta_{1 r}^{2}(x)\right.\right. \\
& \left.\left.+\eta_{2 r}^{2}(x)\right)+u_{1}\left(\eta_{1 r}^{4}(x)+\eta_{2 r}^{4}(x)\right)+u_{2} \eta_{1 r}^{2}(x) \eta_{2 r}^{2}(x)\right),
\end{aligned}
$$

с точностью до коэффициентов имеет тот же вид, что и (31).

Запишем теперь эффективный гамильтониан для представления $\tau_{3}$, характеризуемого базисными функциями (17). Здесь имеют место следующие инварианты, квадратичные по базисным функциям

$$
\begin{gathered}
I_{4}=\left(\psi_{1}^{\tau_{3}}\right)^{2}+\left(\psi_{2}^{\tau_{3}}\right)^{2}+\left(\psi_{3}^{\tau_{3}}\right)^{2}+\left(\psi_{4}^{\tau_{3}}\right)^{2}, \\
I_{5}=\psi_{1}^{\tau_{3}} \psi_{2}^{\tau_{3}}-\psi_{3}^{\tau_{3}} \psi_{4}^{\tau_{3}}=\frac{1}{2}\left(\left|\psi_{1}^{\tau_{3}}\right|^{2}+\left|\psi_{3}^{\tau_{3}}\right|^{2}+\left|\psi_{2}^{\tau_{3}}\right|^{2}+\left|\psi_{4}^{\tau_{3}}\right|^{2}\right) .
\end{gathered}
$$

Видно, что инвариант $I_{5}$ равен сумме $s_{x}^{2}+s_{y}^{2}$ и является обменным по происхождению. Инвариант $I_{4}$, является по природе релятивистским. Инвариант обменного неоднородного взаимодействия для этого представления имеет вид

$$
I_{e x}^{\tau_{3}}=\frac{\partial \psi_{1}^{\tau_{3}}}{\partial x_{i}} \frac{\partial \psi_{2}^{\tau_{3}}}{\partial x_{i}}-\frac{\partial \psi_{3}^{\tau_{3}}}{\partial x_{i}} \frac{\partial \psi_{4}^{\tau_{3}}}{\partial x_{i}}
$$

и преобразуется к тому же виду через величины $s_{x}, s_{y}$, что и в равенстве (28). Инварианты четвертого порядка, записываются следующим образом

$$
\begin{gathered}
\left(\psi_{1}^{\tau_{3}}\right)^{4}+\left(\psi_{2}^{\tau_{3}}\right)^{4}+\left(\psi_{3}^{\tau_{3}}\right)^{4}+\left(\psi_{4}^{\tau_{3}}\right)^{4}, \quad \psi_{1}^{\tau_{3}} \psi_{2}^{\tau_{3}} \psi_{3}^{\tau_{3}} \psi_{4}^{\tau_{3}}, \\
{\left[\left(\psi_{1}^{\tau_{3}}\right)^{2}+\left(\psi_{2}^{\tau_{3}}\right)^{2}\right]\left[\left(\psi_{3}^{\tau_{3}}\right)^{2}+\left(\psi_{4}^{\tau_{3}}\right)^{2}\right]\left[\left(\psi_{1}^{\tau_{3}} \psi_{2}^{\tau_{3}}\right)^{2}+\left(\psi_{3}^{\tau_{3}} \psi_{4}^{\tau_{3}}\right)^{2}\right] .}
\end{gathered}
$$

Эффективный гамильтониан для рассматриваемого представления принимает вид

$$
\begin{aligned}
H_{e f f}^{\tau_{3}} & =\int d^{d} x\left(\frac{r_{2}}{2}\left[\xi_{1}(x) \xi_{2}(x)-\xi_{3}(x) \xi_{4}(x)\right]\right. \\
& +u_{3}\left[\xi_{1}^{4}(x)+\xi_{2}^{4}(x)+\xi_{3}^{4}(x)+\xi_{4}^{4}(x)\right] \\
& +\frac{1}{2}\left[\left(\frac{\partial \xi_{1}}{\partial x}\right)^{2}+\left(\frac{\partial \xi_{2}}{\partial x}\right)^{2}+\left(\frac{\partial \xi_{3}}{\partial x}\right)^{2}+\left(\frac{\partial \xi_{4}}{\partial x}\right)^{2}\right] \\
& +u_{4} \xi_{1}(x) \xi_{2}(x) \xi_{3}(x) \xi_{4}(x)+u_{6}\left[\xi_{1}^{2}(x)+\xi_{2}^{2}(x)\right]\left[\xi_{3}^{2}(x)\right. \\
& \left.\left.+\xi_{4}^{2}(x)\right]+u_{7}\left[\xi_{1}^{2}(x) \xi_{2}^{2}(x)+\xi_{3}^{2}(x) \xi_{4}^{2}(x)\right]\right)
\end{aligned}
$$

где введены обозначения $\psi_{i}^{\tau_{3}}=\xi_{i}, i=1, \ldots, 4$. Этот же вид будет иметь эффективный гамильтониан, если считать, что при преобразованиях симметрии преобразуются коэффициены разложения в равенстве $(21)$, причем в этом случае величины $\xi_{i}=\eta_{i 3}, i=1, \ldots, 4$. Справедливости ради нужно отметить, что для установления обменной природы инварианта $I_{5}$ необходимо явным образом знать базисные функции (17). Вводя теперь обозначения

$$
\begin{array}{ll}
\psi_{1}^{\tau_{3}}=Q_{1}+i \bar{Q}_{1}, & \psi_{2}^{\tau_{3}}=Q_{1}-i \bar{Q}_{1}, \\
\psi_{3}^{\tau_{3}}=Q_{2}+i \bar{Q}_{2}, & \psi_{4}^{\tau_{3}}=i \bar{Q}_{2}-Q_{2},
\end{array}
$$

первое слагаемое в равенстве (37) сводится к обычному виду $Q_{1}^{2}+\bar{Q}_{1}^{2}+Q_{2}^{2}+\bar{Q}_{2}^{2}$ суммы квадратов компонент параметра порядка. В этих переменных эффективный гамильтониан (37) запишется в виде

$$
\begin{aligned}
& H_{\text {eff }}^{\tau_{3}}=\int d^{d} x\left(\frac{r_{2}}{2}\left[Q_{1}^{2}+\bar{Q}_{1}^{2}+Q_{2}^{2}+\bar{Q}_{2}^{2}\right]+u_{3}^{\prime}\left[\left(Q_{1}^{2}-\bar{Q}_{1}^{2}\right)^{2}\right.\right. \\
& \left.+\left(Q_{2}^{2}-\bar{Q}_{2}^{2}\right)^{2}\right]+u_{4}\left[\left(Q_{1}^{2}+\bar{Q}_{1}^{2}\right)\left(Q_{2}^{2}+\bar{Q}_{2}^{2}\right)\right]+u_{6}^{\prime}\left(Q_{1}^{2}-\bar{Q}_{1}^{2}\right) \\
& \times\left(Q_{2}^{2}-\bar{Q}_{2}^{2}\right)+u_{7}\left[\left(Q_{1}^{2}+\bar{Q}_{1}^{2}\right)^{2}+\left(Q_{2}^{2}+\bar{Q}_{2}^{2}\right)^{2}\right] \\
& \left.+\frac{1}{2}\left[\left(\frac{\partial Q_{1}}{\partial x_{i}}\right)^{2}+\left(\frac{\partial \bar{Q}_{1}}{\partial x_{i}}\right)^{2}+\left(\frac{\partial Q_{2}}{\partial x_{i}}\right)^{2}+\left(\frac{\partial \bar{Q}_{1}}{\partial x_{i}}\right)^{2}\right]\right),
\end{aligned}
$$

где $u_{3}^{\prime}=2 u_{3}, u_{6}^{\prime}=4 u_{6}$.

Рассмотрим далее представление $D^{\{k\} \tau_{4}}$ с базисными функциями (20), в котором вектор плотности магнитного момента лежит в плоскости, перпендикулярной главной оси кристалла. Выражения для базисных функций указывают на то, что помимо длинноволновых изменений магнитной структуры имеются также осцилляции 
атомного масштаба вдоль главной оси, определяемые множителем $\cos \left(\pi z / \tau_{z}\right)$. Рассмотрим сначала инварианты второго порядка, используя для базисных функций представление

$$
\begin{array}{ll}
\psi_{1}^{\prime \tau_{4}}=\phi_{3}+i \bar{\phi}_{3}, & \psi_{2}^{\prime \tau_{4}}=\phi_{3}-i \bar{\phi}_{3}, \\
\psi_{3}^{\prime \tau_{4}}=\phi_{4}+i \bar{\phi}_{4}, & \psi_{4}^{\prime \tau_{4}}=i \bar{\phi}_{4}-\phi_{4} .
\end{array}
$$

В этом представлении инвариант второго порядка, который можно получить из инварианта $I_{5}$ простой заменой индекса представления, равен

$$
\left(\phi_{3}^{2}+\bar{\phi}_{3}^{2}+\phi_{4}^{2}+\bar{\phi}_{4}^{2}=\left(s_{y}^{2}+s_{x}^{2}\right)\left(\cos \pi z / \tau_{z}\right)^{2} .\right.
$$

Выражение (41) инвариантно относительно поворотов в спиновом пространстве на любой угол, то есть представляет собой обменный инвариант. Другие возможные инварианты второго порядка являются релятивистскими. Инвариантом четвертого порядка помимо квадрата рассмотренного выше выражения является и величина $\left(\phi_{3}^{2}+\bar{\phi}_{3}^{2}\right)\left(\phi_{4}^{2}+\bar{\phi}_{4}^{2}\right)=s_{x}^{2} s_{y}^{2}\left(\cos \pi z / \tau_{z}\right)^{2}$. Инвариант Лифшица

$$
\begin{aligned}
I_{L}^{\prime \tau_{4}} & =\frac{\partial \psi_{1}^{\prime \tau_{4}}}{\partial x} \psi_{2}^{\prime \tau_{4}}-\psi_{1}^{\prime \tau_{4}} \frac{\partial \psi_{2}^{\prime \tau_{4}}}{\partial x}+\psi_{3}^{\prime \tau_{4}} \frac{\partial \psi_{4}^{\prime \tau_{4}}}{\partial y}-\frac{\partial \psi_{3}^{\prime \tau_{4}}}{\partial y} \psi_{4}^{\prime \tau_{4}} \\
& =i\left(k_{x} s_{x}^{2}+k_{y}^{\prime} s_{y}^{2}\right) \cos \left(\pi z / \tau_{z}\right)
\end{aligned}
$$

имеет релятивистскую природу. Вблизи точки фазового перехода, где существенны спиновые флуктуации, эффективный гамильтониан равен

$$
\begin{aligned}
& H_{e f f}^{\prime \tau_{4}}=\int d^{d} x\left\{\alpha\left(\phi_{3}^{2}+\bar{\phi}_{3}^{2}+\phi_{4}^{2}+\bar{\phi}_{4}^{2}\right)+u_{8}\left(\phi_{3}^{2}+\bar{\phi}_{3}^{2}\right.\right. \\
& \left.+\phi_{4}^{2}+\bar{\phi}_{4}^{2}\right)^{2}+u_{9}\left(\phi_{3}^{2}+\bar{\phi}_{3}^{2}\right)\left(\phi_{4}^{2}+\bar{\phi}_{4}^{2}\right)+\frac{1}{2}\left(\left(\partial \phi_{3} / \partial x_{i}\right)^{2}\right. \\
& \left.\left.+\left(\partial \bar{\phi}_{3} / \partial x_{i}\right)^{2}+\left(\partial \phi_{4} / \partial x_{i}\right)^{2}+\left(\partial \bar{\phi}_{4} / \partial x_{i}\right)^{2}\right)\right\}
\end{aligned}
$$

Отметим, что вид этого эффективного гамильтониана с точностью до обозначения констант совпадает с выражением (5) в работе [3].

Представление $\tau_{4}$, описываемое базисными функциями (18), имеет инвариант

$$
\begin{aligned}
I_{5}^{\prime} & =\psi_{1}^{\tau_{4}} \psi_{2}^{\tau_{4}}-\psi_{3}^{\tau_{4}} \psi_{4}^{\tau_{4}} \\
& =\frac{1}{2}\left(\left|\psi_{1}^{\tau_{4}}\right|^{2}+\left|\psi_{3}^{\tau_{4}}\right|^{2}+\left|\psi_{2}^{\tau_{4}}\right|^{2}+\left|\psi_{4}^{\tau_{4}}\right|^{2}\right)=-2 s_{z}^{2} .
\end{aligned}
$$

В подходе Дзялошинского [16] в этом случае нужно снова вводить функции (25). Обменный инвариант теперь также равен

$$
2 \mathbf{S}^{2}=\frac{1}{2} S^{2}\left(\left|\varphi_{1 \tau_{1}}\right|^{2}+\left|\varphi_{2 \tau_{1}}\right|^{2}+\left|\varphi_{3 \tau_{1}}\right|^{2}+\left|\varphi_{4 \tau_{1}}\right|^{2}\right),
$$

то есть имеет тот же вид, что и инвариант $I_{5}^{\prime}$. Следовательно, этот последний инвариант содержит вклад, который имеет обменную природу. Ясно, что в этом случае реализуется ситуация изинговского магнетика, когда спины ионов железа ориентированы только вдоль оси z кристалла. Инвариант четвертого порядка пропорционален $s_{z}^{4}$. Инвариант Лифшица

$$
\begin{aligned}
I_{L}^{\tau_{4}} & =\frac{\partial \psi_{1}^{\tau_{4}}}{\partial x} \psi_{2}^{\tau_{4}}-\psi_{1}^{\tau_{4}} \frac{\partial \psi_{2}^{\tau_{4}}}{\partial x}+\psi_{3}^{\tau_{4}} \frac{\partial \psi_{4}^{\tau_{4}}}{\partial y}-\frac{\partial \psi_{3}^{\tau_{4}}}{\partial y} \psi_{4}^{\tau_{4}} \\
& =-2 i\left(k_{x}+k_{y}^{\prime}\right) s_{z}^{2}
\end{aligned}
$$

является обменным. Это означает, что фазовый переход второго рода в этом случае не может реализоваться, хотя переход первого рода может произойти.

Наконец, для представления $D^{\{k\} \tau_{3}}$ с базисными функциями (19) запишем их следующим образом

$$
\begin{gathered}
\psi_{1}^{\prime \tau_{3}}=\phi_{5}+i \bar{\phi}_{5}, \quad \psi_{2}^{\prime \tau_{3}}=-\phi_{5}+i \bar{\phi}_{5}, \\
\psi_{3}^{\prime \tau_{3}}=-\phi_{6}-i \bar{\phi}_{6}, \quad \psi_{4}^{\prime \tau_{3}}=-\phi_{6}+i \bar{\phi}_{6} .
\end{gathered}
$$

Базисные функции (19) снова указывают на то, что помимо длинноволновых изменений магнитной структуры возможны еще осцилляции атомного масштаба, задаваемые множителем $\cos \left(\pi z / \tau_{z}\right)$. Инвариант $I_{5}^{\prime} \mathrm{c}$ учетом (19) равен $-2 s_{z}^{2}\left(\cos \pi z / \tau_{z}\right)^{2}$, то есть снова изинговского типа. Инвариантом четвертого порядка помимо квадрата последнего выражения будет инвариант, равный произведению базисных функций, выражение для которого имеет вид

$$
\left(\phi_{5}^{2}+\bar{\phi}_{5}^{2}\right)\left(\phi_{6}^{2}+\bar{\phi}_{6}^{2}\right)=-s_{z}^{4}\left(\cos \pi z / \tau_{z}\right)^{2} .
$$

Вводя обозначение $s_{z} \cos \pi z / \tau_{z}=s_{z}^{\prime}$, инвариант, учитывающий неоднородность распределения спиновой плотности

$$
\left(\partial \phi_{5} / \partial x_{i}\right)^{2}+\left(\partial \bar{\phi}_{5} / \partial x_{i}\right)^{2}+\left(\partial \phi_{6} / \partial x_{i}\right)^{2}+\left(\partial \bar{\phi}_{6} / \partial x_{i}\right)^{2},
$$

где $i=1,2,3$, запишется в виде

$$
\frac{\partial s_{z}^{\prime}}{\partial x_{i}} \frac{\partial s_{z}^{\prime}}{\partial x_{i}}
$$

Инвариант Лифшица

$$
\begin{aligned}
I_{L}^{\prime \tau_{3}} & =\frac{\partial \psi_{1}^{\prime \tau_{3}}}{\partial x} \psi_{2}^{\prime \tau_{3}}-\psi_{1}^{\prime \tau_{3}} \frac{\partial \psi_{2}^{\prime \tau_{3}}}{\partial x}+\psi_{3}^{\prime \tau_{3}} \frac{\partial \psi_{4}^{\prime \tau_{3}}}{\partial y}-\frac{\partial \psi_{3}^{\prime \tau_{3}}}{\partial y} \psi_{4}^{\prime \tau_{3}} \\
& =-2 i\left(k_{x}+k_{y}^{\prime}\right) s_{z}^{2}\left(\cos \pi z / \tau_{z}\right)^{2}
\end{aligned}
$$

имеет обменный характер. Поэтому в данном случае может происходить только переход первого рода.

Дадим еще одну трактовку, обменной природы инвариантов $I_{3}, I_{5}, I_{5}^{\prime}$, приведенных выше. Для представления $V^{\prime}(g) \otimes D^{\{k\} \tau_{1}}$ обменный инвариант, как мы уже писали, имеет вид $2 \mathbf{S}^{2}=\left(\mathbf{S}_{11} \mathbf{S}_{12}+\mathbf{S}_{13} \mathbf{S}_{14}\right)=2\left(s_{x}^{2}+s_{y}^{2}+s_{z}^{2}\right)$. Проектирование представления $V^{\prime}(g) \otimes D^{\{k\} \tau_{1}}$ на $D^{\{k\} \tau_{2}}$ вырезает из этого инварианта величину $\left(s_{x}^{2}+s_{y}^{2}\right)=-I_{3}$. Проектирование $V^{\prime}(g) \otimes D^{\{k\} \tau_{1}}$ на представление $D^{\{k\} \tau_{3}}$ 
также вырезает из написанного инварианта $2 \mathbf{S}^{2}$ сумму $\left(s_{x}^{2}+s_{y}^{2}\right)=I_{5}$. Наконец, проектирование $V^{\prime}(g) \otimes D^{\{k\} \tau_{1}}$ на $D^{\{k\} \tau_{4}}$ вырезает из $2 \mathbf{S}^{2}$ величину $2 s_{z}^{2}$. Сумма вырезанных величин как раз и дает обменный инвариант для представления $V^{\prime}(g) \otimes D^{\{k\} \tau_{1}}$, что и указывает на обменный характер $I_{3}, I_{5}, I_{5}^{\prime}$ инвариантов.

\section{5. Ренормгрупповой анализ фазовых переходов}

Выше уже упоминалось, что в теории Ландау переход второго рода реализуется по одному неприводимому представлению. Проанализируем поэтому сначала возможные переходы в рассматриваемой системе на основе этого предположения. Начнем с представления $D^{\{k\} \tau_{2}}$. Заметим, что в эффективном гамильтониане (31) были в основном учтены инварианты обменной природы, за исключением инварианта $I_{4}$, который фактически учитывает анизотропию системы. Кроме того, слагаемое, принимающее во внимание неоднородное распределение спиновой плотности, вблизи критической точки описывает спиновые флуктуации. Результаты анализа фазового перехода на основе этого эффективного гамильтониана известны, как на основе теории Ландау $(d=3$ в равенстве (31)), так и на основе ренормгруппового подхода $[19,20]$. Поэтому для сравнения с результатами работы [3] опишем здесь результаты ренормгруппового анализа. Вводя величину $y=2+u_{2} / u_{1}$, можно показать [19], что точка $y=2\left(u_{2}=0\right)$ является устойчивой критической точкой, в которой реализуется переход второго рода. Если до перехода выполняется условие $0<y<2\left(-2 u_{1}<u_{2}<0\right)$, то происходит переход в структуру, где $s_{x}=\mp s_{y}$, то есть в системе одновременно сосуществуют волны спиновой плотности с волновыми векторами вдоль осей $X$ и $Y$ кристалла. В том случае, когда значения параметра $y$ лежат в интервале $2<y<6$ $\left(0<u_{2}<4\right)$, то возникает в результате перехода второго рода либо структура $s_{x} \neq 0, s_{y}=0$, либо структура $s_{x}=0, s_{y} \neq 0$. Таким образом, в рассматриваемой ситуации появляется продольная спиновая вдоль оси $X$ или $Y$ соответственно. Отметим, что интервалы значений параметра $u_{2}$, для появления разных фаз, приведенные в данной работе, и в работе [3] противоположны друг другу. В чисто обменном приближении, когда параметр $u_{2}$ равен нулю, продольные волны спиновой плотности с волновым вектором вдоль разных осей оказываются независимыми. Отметим, что такая высокая симметрия эффективного гамильтониана возникает в этом случае за счет спиновых флуктуаций $[19,20]$. Переходы, описываемые на основе эффективного гамильтониана (39) проще всего проанализировать, если выполняются условия для коэффициентов в релятивистских по природе слагаемых в эффективном гамильтониане, $u_{4} \gg u_{3}^{\prime}, u_{6}^{\prime}$, поскольку в этом случае (39) сводится к виду (31). Теперь две поперечные спиновые волны, для которых $s_{x}=\mp s_{y}$, возникают, если $0<u_{4}<2 u_{7}$. Существование же волны, для которой $s_{x} \neq 0, s_{y}=0$, либо $s_{x}=0, s_{y} \neq 0$ имеет место, если $2 u_{7}<u_{4}<6 u_{7}$.

Вопрос о том для каких волн, продольных или поперечных, произойдет переход фактически определяется выписанными выше условиями для коэффициентов эффективных гамильтонианов. Если любое из этих условий реализуется для продольных волн, то произойдет первый переход, если эти условия реализуются для поперечных волн, то ниже перехода возникнут волны поперечной спиновой плотности. Отметим, что за пределами области спиновых флуктуаций в эффективный гамильтониан системы необходимо включить инвариант Лифшица.

Ренормгрупповой анализ фазового перехода для эффективного гамильтониана (42) проведен в работе [3]. В этой работе установлено, что для реализации структуры с волнами спиновой плотности с волновыми векторами $\mathbf{k}$ и $\mathbf{k}^{\prime}$ необходимо выполнение условия $u_{9}>0$, а для существования одной волны должно выполняться противоположное неравенство. При этом в направлении оси $z$ на волны налагается также простая осцилляция, определяемая множителем $\cos \pi z / \tau_{z}$. Отметим еще раз, что изменение направления магнитных моментов вдоль оси $Z$ происходит на атомных масштабах.

Пока мы рассмотрели только ситуацию с ориентацией магнитных моментов в плоскости $X Y$ кристалла. Две последние структуры, допускаемые симметрией, приводят к ориентации спиновых моментов вдоль главной оси кристалла. Этот означает, что в системе в этом случае могут реализуются структуры изинговского типа, а, следовательно, в системе должна существовать одноосная магнитная анизотропия, превышающую по величине анизотропию в плоскости слоев. Однако взаимодействия магнитных моментов внутри слоев обычно превышает межслоевое взаимодействие, поэтому реализация таких структур в дигерманиде железа маловероятна. Выше уже упоминалось, что такие структуры могут возникать только в результате переходов первого рода.

Во всех трех случаях рассмотренных фазовых переходов второго рода в предположении переходов по одному неприводимому представлению спиральные структуры не реализуются. Могут появиться только поперечные или продольные волны спиновой плотности. Для описания спиральных магнитных структур необходимо использовать базисные функции (16) и (17), которые относятся к разным неприводимым представлениям пространственной группы. Отсюда можно сделать вывод, что для возникновения спиральных структур переход в несоизмеримую фазу должен происходить по двум представлениям. В большинстве работ, кроме того, утверждалось, что переход в несоизмеримую магнитную фазу происходит путем одного фазового перехода при температуре $290 \mathrm{~K}$. Только в одной работе [9] на основе аномалий в рассеяния ультразвука в окрестности этой температуры обнаружены два перехода, отстоящие по температуре на $0.4 \mathrm{~K}$. Тогда оставаясь на позициях теории Ландау можно считать, что в дигерманиде железа 
происходят два фазовых перехода, связанные с представлениями $\tau_{2}$ и $\tau_{3}$, но они слабо сдвинуты по температуре. Поэтому в случае переходов второго рода ввиду развитых спиновых флуктуаций можно для упрощения анализа задачи предположить, что оба перехода имеют одинаковую температуру. Проанализируем эту ситуацию в обменном приближении. В этом случае эффективный гамильтониан системы имеет вид

$$
H=H_{e x}^{\tau_{2}}+H_{e x}^{\tau_{3}}+H_{i n t},
$$

где

$$
\begin{gathered}
H_{e x}^{\tau_{2}}=\int d^{d} x\left(\frac{r}{2}\left[s_{x}^{2}+s_{y}^{2}\right]+u_{1}\left[s_{x}^{2}+s_{y}^{2}\right]^{2}\right. \\
\left.+\frac{1}{2}\left[\left(\frac{\partial s_{x}}{\partial x_{i}}\right)^{2}+\left(\frac{\partial s_{y}}{\partial x_{i}}\right)^{2}\right]\right), \\
H_{e x}^{\tau_{3}}=\int d^{d} x\left(\frac{r}{2}\left[\left(s_{x}^{\prime}\right)^{2}+\left(s_{y}^{\prime}\right)^{2}\right]+u_{2}^{\prime}\left[\left(s_{x}^{\prime}\right)^{2}+\left(s_{y}^{\prime}\right)^{2}\right]^{2}\right. \\
\left.+\frac{1}{2}\left[\left(\frac{\partial s_{x}^{\prime}}{\partial x_{i}}\right)^{2}+\left(\frac{\partial s_{y}^{\prime}}{\partial x_{i}}\right)^{2}\right]\right), \\
H_{i n t}=\int d^{d} x u_{3}^{\prime}\left[s_{x}^{2}+s_{y}^{2}\right]\left[\left(s_{x}^{\prime}\right)^{2}+\left(s_{y}^{\prime}\right)^{2}\right] .
\end{gathered}
$$

Во втором из равенств (49) параметр $u_{2}^{\prime}=u_{7}$. Заметим, что в обменном приближении в гамильтониане взаимодействия других слагаемых нет. Тогда исследуемая задача полностью совпадает с таковой, рассмотренной в работе [21]. В этой работе показано, что для нашего случая имеется критическая неподвижная точка, равная

$$
u_{1}=u_{2}^{\prime}=\frac{u_{3}^{\prime}}{2}=\frac{\varepsilon}{96 \pi^{2}} .
$$

Таким образом, в системе имеет место фазовый переход второго рода. Этот результат не совпадает с выводами работы [3], в которой такой переход может быть только первого рода. Полученный нами результат согласуется с экспериментальными данными относительно типа перехода. В результате такого перехода в системе может появиться спиральная структура. Возможность появление такой структуры показать, используя разложения (4) и (6).

\section{6. Критическая динамика перехода второго рода}

Вернемся снова к ситуации, когда переход в несоизмеримую структуру происходит по одному представлению. Пусть это будет для определенности переход по представлению $D^{\{k\} \tau_{2}}$. В статическом случае в точке фазового перехода имеет место соотношение $[19,20] u_{2}=0$ для коэффициентов в гамильтониане (31),что приводит к повышению симметрии эффективного гамильтониана. Это позволяет проанализировать критическую динамику этого перехода.
Выпишем сначала статическое действие $S^{s t, \tau_{2}}$ нашей модели для перехода по этому представлению. В этом действии учтем наличие внешнего магнитного поля $h_{0 m}$ и намагниченности $m$, которая будет играть роль сохраняющейся величины. Введем переменные

$$
\Psi=s_{x}+i s_{y}, \quad \Psi^{+}=s_{x}-i s_{y} .
$$

В этом случае статическое действие можно записать в виде, прямо совпадающим со статическим действием модели $F$ критической динамики [22]

$$
\begin{aligned}
S^{s t, \tau_{2}}= & \int d^{d} x\left(-\frac{\partial \Psi^{+}}{\partial x} \frac{\partial \Psi}{\partial x}-\frac{u_{i s t}\left(\Psi^{+} \Psi\right)^{2}}{6}\right. \\
& \left.+u_{2 \Psi} m \Psi^{+} \Psi-\frac{m^{2}}{2}+m h_{0 m}\right),
\end{aligned}
$$

где $u_{1 s t}=6 u_{1}$, а $u_{2} \Psi-$ новый параметр взаимодействия с сохраняющимся магнитным моментом. Намагниченность $\mathrm{m}$ считается вещественной величиной. Просто показать, что динамическое действие этой модели, сопровождающееся введением двух дополнительных комплексных полей и одного вещественного поля, является перенормируемым [22], то есть для анализа этой модели применим ренормгрупповой подход. Полный анализ модели $F$ на основе этого подхода проведен в работе [23]. Найдена область стабильности динамической скейлинговой фиксированной точки. Таким образом, и критическая динамика перехода в несоизмеримую магнитную структуру типа продольной спиновой волны содержит динамическую фиксированную точку. Динамика перехода в структуру с поперечной волной спиновой плотности в случае выполнения условия $u_{4} \gg u_{3}^{\prime}, u_{6}^{\prime}$ также будет содержать динамическую фиксированную точку.

\section{7. Заключение}

В работе на основе симметрийного подхода проведено описание возможных магнитных структур несоизмеримой магнитной фазы соединения $\mathrm{FeGe}_{2}$ и проанализированы фазовые переходы, в результате которых эти фазы могу реализоваться.

Для описания магнитных структур использованы два подхода. Первый из них основан на магнитном представлении пространственной группы [10], а второй на разложении вектора плотности магнитного момента в несоизмеримой фазе по базисным функциям неприводимых представлений пространственной группы парамагнитной фазы $[15,16]$ с учетом того, что в выражения для энергии спиновые переменные могут входить только в четных степенях. Было показано, что при использовании во втором подходе для многолучевой звезды волнового вектора неприводимых представлений полной пространственной группы оба подхода при описании магнитных структур дают одинаковые результаты. Однако первый подход технически 
оказывается проще, так как для своего использования требует знания только малых неприводимых представлений для нулевого блока группы волнового вектора. Во втором же подходе необходимо определять базисные функции тех неприводимых представлений полной пространственной группы, фигурирующих в разложении (21), которые содержатся в приводимых представлениях, представляющих собой прямое произведение псевдовекторного и неприводимого представления пространственной группы, входящих в разложение (8). Отметим, что оба набора найденных неприводимых представлений в первом и во втором подходе полностью совпадают.

Показано, что структуры типа продольных спиновых волн связаны с представлением $\tau_{2}$ пространственной группы $I 4 / \mathrm{mcm}\left(D_{4 h}^{18}\right)$ для волнового вектора $\mathbf{k}=(2 \pi \mu / \tau, 0,0), 2 \mu \neq 0, \pm 1, \pm 2, \ldots$, тогда как представление $\tau_{3}$ для этого же волнового вектора приводит к появлению структур типа поперечных спиновых волн. При определенных соотношениях между компонентами параметров порядка в системе могут формироваться плоские спиральнные магнитные структуры. Установлено также, что в системе могут появиться волны поперечной спиновой плотности, содержащие модуляцию вдоль главной оси кристалла атомного масштаба. Такая структура связана с представлением $\tau_{4}$. Симметрийный анализ допускает также возможность существования волн поперечной спиновой плотности, когда вектор плотности магнитного момента ориентирован вдоль главной оси кристалла. Такая структура связана также с представлениями $\tau_{3}$ или $\tau_{4}$. При этом в структуpe, связанной с $\tau_{3}$ имеется дополнительная модуляция атомного масштаба вдоль главной оси. Отметим, что в первом подходе модуляции атомного масштаба выявить не удается.

Построение эффективного гамильтониана, с помощью которого описывается переход в допустимую симметрией магнитную структуру, осуществлялся на основе построения инвариантных величин относительно их преобразований по неприводимому представлению полной пространственной группы, по которому переход происходит. В первом подходе компонентами параметра порядка, преобразующимися по этому представлению, являются коэффициенты разложения плотности магнитного момента по базисным функциям магнитного представления пространственной группы [10]. Во втором подходе эти инварианты строились на основе базисных функций самого неприводимого представления, входящего в разложение (21). Выше было показано, что в принципе оба подхода приводят к одинаковым выражениям для эффективных гамильтонианов, если правильно определена физическая природа этих инвариантов. Основная проблема первого подхода на этом этапе состоит в том, что природу этих инвариантов трудно определить непосредственно из выражения этого инварианта. Отметим одну важную деталь, установленную в данной работе. В ссылке [16] было дано выражение для обменного инварианта в виде (26), который помимо спиновой части содержит также вклад от блоховских функций. Однако явный вид базисных функций неприводимых представлений пространственной группы, участвующих в разложении (8), позволил установить еще один обменный инвариант, который строится из функций $\mathbf{S}_{1 \alpha}$ в обозначениях [16], а именно $\mathbf{S}^{2}$, в котором отсутствует вклад от пространственных блоховских функций. Наличие этого инварианта позволяет во втором подходе явным образом определять обменные инварианты, которые можно построить исходя из базисных функций (16)-(18) неприводимых представлений пространственной группы.

Проведен ренормгрупповой анализ перехода из парамагнитного состояния в несоизмеримую магнитную фазу. Анализ проводился сначала в ситуации, когда переход происходит по одному неприводимому представлению. Найдено, что переход в длиннопериодическую структуру как для поперечных, так и продольных спиновых волн, когда вектор плотности магнитного момента лежит в базисной плоскости происходит в результате фазового перехода второго рода. Найдены условия, которым должны удовлетворять параметры эффективного гамильтониана, чтобы реализовались случаи, когда возникает одна волна спиновой плотности с волновым вектором, ориентированным либо вдоль оси $X$, либо вдоль оси $Y$ кристалла, либо обе таких волны в отдельности для поперечных и продольных спиновых волн.

В работе на основе экспериментальных данных по рассеянию нейтронов [3], была рассмотрена ситуация, когда появление несоизмеримого дальнего магнитного порядка вызвано двумя магнитными переходами, один из которых связан с представлением $\tau_{2}$, а второй c $\tau_{3}$. Предполагалось, что оба перехода происходят при одинаковой температуре. Различием температур этих переходов мы пренебрегли ввиду малости этого различия $(0.4 \mathrm{~K})$, а также основываясь на том, что во многих работах это различие не обнаружено. Показано, что и в этом случае в отличие от теоретического рассмотрения этой ситуации в работе [3], имеет место переход второго рода с устойчивой точкой, задаваемой равенством (47). Следует обратить внимание на то обстоятельство, что на критической поверхности для параметров эффективных гамильтонианов при инвариантах четвертого порядка должно выполняться равенство $u_{1}=u_{2}^{\prime}$, что является жестким ограничением на условия реализации такого перехода.

Из результатов работ $[19,20]$, следует, что в точке перехода для продольных спиновых волн симметрия системы становится выше исходной симметрии эффективного гамильтониана. В этом случае критическую динамику этого перехода можно описать на основе $F$-модели указанной динамики, поскольку статическое действие в точке перехода совпадает с таковым для указанной модели. 


\section{Список литературы}

[1] Ю.А. Изюмов, Э.З. Курмаев. Высокотемпературные сверхпроводники на основе FeAs-соединений. НИЦ „Регулярная и хаотическая динамика“, М.-Ижевск (2010). 334 с.

[2] E. Kren, P. Sabo. Phys. Lett. 11, 215 (1964).

[3] L.M. Corliss, J.M. Hasting, W. Kunnmann, R. Tomas, J. Zhuang. Phys. Rev. B 31, 4337 (1985).

[4] Ю.А. Дорофеев, А.З. Меньшиков, Г.А. Будрина, В.Н. Сыромятников. ФММ 63, 1110 (1987).

[5] Р.П. Кренцис, А.В. Михельсон, П.В. Гельд. ФТТ 12, 2470 (1970).

[6] К.Б. Власов, Е.В. Устелемова, Р.И. Зайнуллина, М.А. Миляев, С.В. Устелемов. ФТТ 32, 1385 (1990).

[7] P.D. Babu, P.K. Mishra, V. Dubc, R. Mishra, P.U. Sastry, G. Ravikumar. AIP Conf. Proc. 1591, 1586 (2014).

[8] G.E. Gerchnev, A.A. Lyogenkaya, V.B. Pluzhnikov, A.V. Fedorchenko. Low Temp. Phys. 40, 384 (2014).

[9] V.B. Pluzhnikov, D. Feder, E. Fawcett. J. Magn. Magn. Matter. 27, 343 (1982).

[10] Ю.А. Изюмов, В.Е. Найш, Р.П. Озеров. Нейтронография магнетиков. Атомиздат, М. (1981). 311 с.

[11] H.J. Wallaum. Z. Metallknd. 35, 218 (1943).

[12] J.B. Forsgth, C.E. Johnson, P.J. Brown. Phil. Mag. 10, 713 (1964).

[13] О.В. Ковалев, Неприводимые индуцированные представления и копредставления федоровских групп. Наука, М. (1986). $367 \mathrm{c}$.

[14] Г.Л. Бир, Г.Е. Пикус. Симметрия и деформационные эффекты в полупроводниках, Наука, М. (1972). 584 с.

[15] О.В. Ковалев. ФТТ 5, 3156 (1963).

[16] И.Е. Дзялошинский. ЖЭТФ 46, 1420 (1964).

[17] C.J. Bradley, A.P. Cracknell. The Mathematical Theory of Symmetry in Solids. Representation Theory for Point Groups and Space Groups. Clarendon Press, Oxford (1972). 745 p.

[18] Л.Д. Ландау, Е.М. Лифшиц. Статистическая физика. Наука, М. (1976). Ч. 1. $583 \mathrm{c}$.

[19] K.G. Wilson, M. Fisher. Phys. Rev. Lett. 28, 240 (1972).

[20] В.В. Меньшенин, ЖЭТФ 147, 1179 (2015).

[21] Е.Е. Городецкий. В.М. Запрудский. ЖЭТФ 69, 1013 (1975).

[22] А.Н. Васильев. Квантовополевая ренормгруппа в теории критического поведения и стохастической динамики. ПИЯФ, СПб. (1998). 773 с.

[23] De Dominicis, L. Peliti. Phys. Rev. B 18, 353 (1978).

Редактор Ю.Э. Китаев 